\title{
Análise de custo da assistência de crianças e adolescentes com condições crônicas complexas
}

\author{
Analysis of the cost of care for children and adolescents with \\ medical complex chronic conditions
}

Márcia Pinto (https://orcid.org/0000-0001-7568-5014) ${ }^{1}$

Romeu Gomes (https://orcid.org/0000-0003-3100-8091) ${ }^{1}$

Roberta Falcão Tanabe (http://orcid.org/0000-0002-6862-1597) ${ }^{1}$

Ana Carolina Carioca da Costa (https://orcid.org/0000-0002-9456-3319) ${ }^{1}$

Martha Cristina Nunes Moreira (https://orcid.org/0000-0002-7199-3797) ${ }^{1}$

\footnotetext{
${ }^{1}$ Instituto Nacional de Saúde da Mulher, da Criança e do Adolescente Fernandes Figueira (IFF/Fiocruz), Fundação Oswaldo Cruz. Av. Rui Barbosa 716, Flamengo. 20021-140 Rio de Janeiro RJ Brasil. mpinto@iff.fiocruz.br
}

\begin{abstract}
This paper aimed to identify the use of technology and to analyze the cost of hospital care for children and adolescents with medical complex chronic conditions at a public federal hospital specialized in high-complexity pediatric care, and was performed concomitantly with a prospective cohort study conducted over a one-year period. It included 146 patients with complex medical chronic conditions and 37 nonchronic patients. The analysis showed that most patients had, on average, two hospitalizations a year and were diagnosed with diseases related to at least two organic systems. Catheters, drains and gastrostomy were the most common technologies used. Median direct costs of patients with medically complex chronic conditions were higher than those of non-chronic patients when comparing the use of technology. The study shows high hospitalization cost to these patients. Technology use and hospitalization care costs documentation yields more data to support decision-makers in the planning, managing, and financing of pediatric health policies.

Key words Child, Youth, Chronic disease, Cost and cost analysis.
\end{abstract}

Resumo O objetivo deste artigo foi identificar a utilização de tecnologias e estimar o custo direto da atenção hospitalar de crianças e adolescentes com condições crônicas complexas em um hospital público federal especializado na assistência de alta complexidade a pacientes pediátricos. O trabalho foi realizado concomitantemente com um estudo de coorte durante o período de um ano e incluiu 146 pacientes com condições complexas crônicas e 37 pacientes não-crônicos. A análise identificou que a maioria dos pacientes com condições complexas crônicas internou em média duas vezes em um ano e que tinham doenças com o envolvimento de pelo menos dois sistemas orgânicos. O uso de drenos e cateteres e a gastrostomia foram as tecnologias de maior utilização. Na comparação com os pacientes não-crônicos, o custo direto mediano dos pacientes com condições complexas crônicas foi superior quando se comparou a utilização de tecnologias. O estudo indica um elevado custo da atenção hospitalar para esses pacientes. Documentar a utilização de tecnologias e o custo da atenção hospitalar permite subsidiar os gestores e contribuir para a tomada de decisões de planejamento, gestão e financiamento das políticas de saúde na área pediátrica.

Palavras-chave Criança, Adolescente, Custo $e$ análise de custos. 


\section{Introdução}

Em 2003, a Organização Mundial de Saúde associou o reconhecimento das transformações no cenário epidemiológico à necessidade de reorganização dos sistemas de saúde considerando a atenção e cuidado às pessoas com condições de saúde crônicas ${ }^{1}$. No interior desse grupo urge reconhecer as necessidades de crianças e adolescentes, cuja situação de cronicidade cursa com complexidades variadas que se caracterizam por limitações de funções físicas e/ou mentais, cuidado multiprofissional que demanda articulação entre diversas áreas da saúde, com especial destaque para a reabilitação, dependência de múltiplas medicações e de tecnologias para alimentação, respiração e excreção ${ }^{2-4}$. Esses grupos são reconhecidos na literatura por diversas definições - Crianças com Necessidades Especiais de Saúde, Crianças Dependentes de Tecnologia, Crianças com Condições Médicas Complexass ${ }^{2,4-6}$. Neste artigo, utilizamos o termo Condição Crônica Complexa de Saúde (CCC) para categorizar crianças e adolescentes e que permite uma síntese das situações que a vida com a cronicidade deflagra: duração de sintomas que supera 12 meses compromete ao mesmo tempo um ou mais sistemas orgânicos, e demanda cuidado pediátrico especializado em centros de atenção terciária.

As CCC são irreversíveis e representam um alto custo para os pacientes e suas famílias, requerendo adaptações familiares e na comunidade e um sistema de saúde eficiente $e^{3,4,7-10}$. No interior da categoria "condições crônicas" existe o grupo de crianças e adolescentes com CCC cuja prevalência está crescendo em virtude da redução da mortalidade infantil, do acesso às novas tecnologias e melhorias nos indicadores sociais e de saúde ${ }^{7,11}$. Em países desenvolvidos, estimase em aproximadamente $16 \%$ na população até 18 anos de idade ${ }^{4}$. Até trinta anos atrás, crianças com essas condições não sobreviviam ${ }^{12}$, mas observa-se que atualmente existe a possibilidade de serem tratadas e sobreviverem com perfis medicamente frágeis e com necessidades de cuidados especiais de saúde ${ }^{3}$.

As crianças e adolescentes com CCC utilizam serviços de saúde intensivos em tecnologia e recursos humanos, através de internações prolongadas e processos de alta hospitalar que demandam muitas negociações com atores situados em outros setores da sociedade - família, Poder Judiciário, serviços de atenção básica e de assistência social ${ }^{13}$. Durante a hospitalização, esses pacientes se caracterizam pela dependência de tecnologia e por uma situação clínica frágil, sujeitos a instabilidades e agravamentos do seu quadro. E, ainda que representem uma parcela menor da população, esse grupo com CCC é responsável por uma significativa proporção dos gastos hospitalares ${ }^{4}$. Nesse sentido, estimar o custo da assistência médica tem sido um objeto de análise em países desenvolvidos $^{14,15}$.

A emergência sanitária em virtude da epidemia pelo vírus Zika no Brasil em 2015 incrementa a importância dessa discussão, considerando que bebês nascidos com microcefalia ou com os agravos variados provocados pela Síndrome da Infecção Congênita pelo vírus Zika farão parte do grupo de crianças que crescem com $\mathrm{CCC}^{16}$. O Ministério da Saúde estima que 29\% dos municípios brasileiros notificaram casos de microcefalia e alterações no sistema nervoso ${ }^{17}$.

O presente artigo contribui para sistematizar e analisar dados sobre o custo da assistência médica de crianças e adolescentes com CCC hospitalizadas e a utilização de tecnologias de saúde. Essas condições demandam uma ampla gama de serviços de saúde que representam um desafio para o Sistema Único de Saúde (SUS). Esses novos e emergentes problemas de saúde, que tocam complexidades múltiplas, convocam uma definição sobre recursos adequados para capacitação de profissionais, programação sobre demandas de tecnologias para suporte a vida, organização de redes de atenção e financiamento adequado ${ }^{12}$.

A literatura brasileira que sistematiza os padrões de utilização de tecnologias e os custos médicos ainda é pouca. Discutir sobre estes padrões no grupo de crianças e adolescentes com CCC torna-se uma questão importante dada a relevância da sua participação no orçamento do setor saúde, tanto em cenários nacionais como internacionais ${ }^{14,18}$. Este estudo faz parte de uma pesquisa mais ampla que sistematizou o perfil de morbidade hospitalar das crianças e adolescentes hospitalizados e seu objetivo foi calcular o custo direto da assistência médica de uma coorte de pacientes hospitalizados com CCC.

\section{Métodos}

Trata-se de uma análise retrospectiva de uma coorte de pacientes com CCC conduzida entre maio/2014 e abril/2015. Foram incluídos todos os pacientes entre 0 e 18 anos hospitalizados durante 12 meses no Instituto Nacional de Saúde da Mulher, da Criança e do Adolescente Fernandes Figueira (IFF), hospital público federal especia- 
lizado em assistência pediátrica, localizado no município do Rio de Janeiro, com abrangência em todo o estado do Rio de Janeiro. A instituição também recebe casos de outras regiões do Brasil por sua qualificação como referência para doenças raras em crianças e adolescentes. Como estas doenças clinicamente podem ser identificadas como CCC, isso faz com que as pesquisas conduzidas nesse ambiente possam vir a refletir demandas nacionais que o mesmo aglutina. $\mathrm{O}$ estudo foi aprovado pelo Comitê de Ética em Pesquisa da instituição.

A definição da condição crônica ou não-crônica da criança foi feita no momento da internação. Se no decorrer da internação algum paciente não-crônico fosse submetido a determinados procedimentos que indicassem dependência de tecnologia como índice de cronicidade, como traqueostomia e gastrostomia, ele seria incluído no grupo de CCC. Os pacientes foram identificados a partir da classificação proposta por Feudtner et al. ${ }^{19}$ e atualizada pela Classificação Internacional de Doenças - 10a Revisão (CID-10). Uma nova versão daquela classificação foi publicada em 2014, adaptando o diagnóstico de CCC à CID-10. Foram acrescentadas três novas categorias: diagnósticos de origem no período neonatal, transplantes e dependência de tecnologia ${ }^{20}$. Esta atualização foi confirmada neste estudo, através de consulta aos especialistas da área pediátrica com experiência na assistência médica às crianças e adolescentes com diagnóstico de CCC.

Os pacientes readmitidos e aqueles transferidos de outros setores do hospital - ambulatório de pediatria geral, ambulatórios especializados, unidades de terapia intensiva neonatal e intensiva pediátrica, enfermarias de cirurgia pediátrica e doenças infecciosas e unidades semi-intensiva e de pacientes internados - foram incluídos no período de acompanhamento da coorte. Assim, os pacientes com CCC foram divididos nos seguintes grupos conforme a CID-10: respiratório, metabólico, neuromuscular, cardiovascular, renal, gastrointestinal, doenças hematológicas, imunodeficiências, transtornos psiquiátricos, deficiências congênitas ou genéticas e câncer.

Em seguida, a partir do perfil clínico, a população do estudo foi subdividida em: i. pacientes que utilizaram tecnologias indicativas de complexidade: traqueostomia, gastrostomia, uso de drenos (tórax, peritoneal ou subgaleal) e cateteres (derivação ventrículo peritoneal, venoso profundo ou de inserção periférica), uso de oxigenioterapia, ventilação mecânica invasiva, fórmulas infantis especiais e antibioticoterapia; ii. número de diagnósticos conforme os grupos da CID-10 associados às CCC; iii. procedência dos pacientes readmitidos ou transferidos de outros setores do hospital; e iv. desfechos clínicos: alta com e sem uso de tecnologia, permanência hospitalar até o final do estudo e óbito hospitalar.

Estimou-se o custo médico direto e adotouse a perspectiva do SUS provedor. O método aplicado foi o do custo por paciente que considera a sua trajetória pelos diferentes serviços. $\mathrm{O}$ sistema de custo por absorção por centro de custo hospitalar permitiu a valoração dos recursos de saúde. Foi calculado o custo direto total por paciente, considerando o valor da diária hospitalar de cada serviço pelo qual o paciente foi atendido. Os itens de custos incluídos foram: exames (laboratoriais e de imagem), materiais, medicamentos, fórmulas lácteas especiais, recursos humanos, uso de oxigenioterapia e administrativos (energia elétrica, telefone, água, limpeza, segurança, coleta de resíduos químicos e ambientais e lavanderia). $\mathrm{O}$ horizonte temporal foi de um ano e não considerou o período anterior e posterior ao início e término da coorte. Não foram aplicados descontos ou ajuste inflacionário devido ao curto período de tempo. O custo refere-se a reais (R\$) de 2016.

Incluiu-se uma comparação do custo entre os pacientes com CCC e os pacientes não-crônicos, a partir da utilização das seguintes tecnologias médicas prescritas para ambos os grupos: fórmulas lácteas especiais, antibioticoterapia, oxigenioterapia, uso de ventilação não-invasiva e exames de imagem (raio-x, tomografia, ultrassonografia e ecocardiografia). A hipótese era que os pacientes com CCC teriam maior consumo de recursos de saúde que os não-crônicos e, portanto, maiores custos.

Para descrição do custo, foram apresentados os valores medianos, mínimos e máximos. As variáveis categóricas foram apresentadas através de frequências absolutas e percentuais. Com o objetivo de verificar o impacto da presença de comorbidades, procedência do paciente, duração das internações e realização de procedimentos sobre o custo total da internação, foram realizadas análises de regressões univariadas utilizando o modelo de equações de estimativas generalizadas (GEE, do inglês Generalized Estimating Equations). Como a variável resposta, neste caso, o custo direto total dos pacientes com CCC não possuía distribuição Normal, foi aplicada a transformação logarítmica para fins de adequação do modelo supracitado. O pressuposto de normalidade foi testado através do teste de Kolmogorov-Smirnov. A significância estatística foi mensurada com 
base em um nível de significância de 5\%. Além do logaritmo, o custo direto total por paciente foi agregado e apresentado, em valores absolutos, como custo mediano direto total da assistência de pacientes com CCC e de pacientes não-crônicos. As análises foram realizadas nos softwares SPSS (SPSS Inc. Release VCSI. SPSS Statistics for Windows, versão 18, IL, EUA) e R (R Core Team. Foundation for Statistical Computing V, Austria, versão 3.2.2).

\section{Resultados}

Durante o período do estudo, foram incluídos 183 pacientes, dos quais $146(79,8 \%)$ com CCC. Pacientes não-crônicos totalizaram 37 indivíduos $(20,2 \%)$. Foram registradas 241 internações. O grupo com CCC registrou idade mediana de um ano e mediana de permanência hospitalar de 11 dias (média: 35 dias; desvio-padrão: 72 dias). A Tabela 1 mostra que do total de pacientes com CCC, $35,7 \%$ tinham doenças com o envolvimento de pelo menos dois sistemas orgânicos, $62,4 \%$ de três sistemas e, aproximadamente, $92 \%$ apresentaram duas internações durante um ano. As hospitalizações mais frequentes foram registradas entre os pacientes com diagnóstico de defeitos genéticos ou congênitos $(78,1 \%)$, doenças respiratórias $(64,4 \%)$ e neuromusculares $(50 \%)$. O uso de dreno e/ou cateter $(39,7 \%)$ e a gastrostomia $(15,8 \%)$ foram as tecnologias mais utilizadas durante a hospitalização.

A Tabela 2 apresenta a comparação entre os pacientes com CCC e os não-crônicos, e o custo mediano total do primeiro grupo foi superior, com variação entre $60 \%$ na internação para o diagnóstico de doenças respiratórias e 657\% para a realização de tomografia. Para os procedimentos realizados durante o tratamento, o custo variou de $133 \%$ a $339 \%$ entre os dois grupos.

Em relação aos pacientes com CCC, os resultados do modelo de regressão evidenciaram que o tempo de permanência, o número de sistemas afetados, o serviço de procedência do paciente, os desfechos e a necessidade de tecnologias na alta hospitalar aumentaram significativamente o custo. $\mathrm{O}$ custo mediano total foi diretamente proporcional à duração da internação hospitalar (Figura 1a) e altamente sensível ao número de doenças/sistemas orgânicos afetados (Figura 1b), sendo o maior custo verificado para os pacientes com CCC com diagnóstico de doenças respiratórias e metabólicas (Figuras 1c e 1d). As demais categorias de doenças classificadas pela CID-10
Tabela 1. Características clínicas e de utilização de tecnologias de pacientes em um hospital público federal, 2016

\begin{tabular}{|c|c|c|}
\hline Características clínicas & $\mathbf{n}$ & $\%$ \\
\hline Condição crônica complexa & 146 & 79,8 \\
\hline Não-crônicos & 37 & 20,2 \\
\hline \multicolumn{3}{|l|}{$\begin{array}{l}\text { Utilização de tecnologias durante a } \\
\text { internação }\end{array}$} \\
\hline Traqueostomia & 7 & 4,8 \\
\hline Gastrostomia & 23 & 15,8 \\
\hline Dreno/cateter & 58 & 39,7 \\
\hline $\begin{array}{l}\text { Shunt ventricular do líquido } \\
\text { cefalorraquidiano }\end{array}$ & 8 & 5,5 \\
\hline \multicolumn{3}{|l|}{ Hospitalizações } \\
\hline Uma & 106 & 72,6 \\
\hline Duas & 28 & 19,2 \\
\hline Três & 8 & 5,5 \\
\hline Quatro & 3 & 2,1 \\
\hline Cinco & 1 & 0,7 \\
\hline \multicolumn{3}{|l|}{ Doenças } \\
\hline Defeitos genéticos ou congênitos & 114 & 78,1 \\
\hline Respiratórias & 94 & 64,4 \\
\hline Neuromusculares & 73 & 50,0 \\
\hline Gastrointestinais & 62 & 42,5 \\
\hline Cardiovasculares & 43 & 29,5 \\
\hline Metabólicas & 27 & 18,5 \\
\hline Renais & 20 & 13,7 \\
\hline $\begin{array}{l}\text { Hematológicas e } \\
\text { imunodeficiências }\end{array}$ & 10 & 6,8 \\
\hline Transtornos psiquiátricos & 1 & 0,7 \\
\hline Câncer & 1 & 0,7 \\
\hline \multicolumn{3}{|l|}{$\begin{array}{l}\text { Diagnósticos associados com CCC } \\
\text { (CID-10) }\end{array}$} \\
\hline $\mathrm{Um}$ & 16 & 11,0 \\
\hline Dois & 36 & 24,7 \\
\hline Três & 39 & 26,7 \\
\hline Quatro & 38 & 26,0 \\
\hline Cinco & 15 & 10,3 \\
\hline Seis & 2 & 1,4 \\
\hline
\end{tabular}

(neuromusculares, cardiovasculares, renais, gastrointestinais, hematológicas ou de deficiência imunológica, distúrbios psiquiátricos e deficiências genéticas ou congênitas e câncer) não apresentaram significância estatística para o custo mediano total.

O custo dos pacientes com CCC oriundos de duas unidades de internação pediátrica - semi-intensiva e de pacientes internados - foi superior ao estimado para os outros setores do hospital. O menor custo foi associado aos pacientes dos ambulatórios especializados quando 
Tabela 2. Custo total mediano de pacientes com condição crônica complexa de saúde e pacientes não-crônicos hospitalizados em um hospital público federal, 2016.

\begin{tabular}{|c|c|c|c|}
\hline \multirow[t]{2}{*}{ Variável } & Pacientes não-crônicos $(\mathrm{R} \$)^{\mathrm{a}}$ & $\begin{array}{l}\text { Pacientes com condição crônica } \\
\text { complexa de saúde }(\mathrm{R} \$)^{\mathrm{a}}\end{array}$ & \multirow[t]{2}{*}{ p-valor } \\
\hline & $(\mathbf{n}=37)$ & $(n=146)$ & \\
\hline \multicolumn{4}{|l|}{ Utilização de tecnologias } \\
\hline Fórmulas lácteas especiais & $18.507,64(2.022,45-40.449,00)$ & $40.449,00(2.022,45-792.562,68)$ & $<0,01$ \\
\hline Antibioticoterapia & $12.134,70(2.022,45-169.834,86)$ & $28.314,30(2.022,45-792.562,68)$ & $<0,01$ \\
\hline Oxigenioterapia & $12.134,70(2.022,45-169.834,86)$ & $31.421,19(2.022,45-762.079,50)$ & $<0,01$ \\
\hline $\begin{array}{l}\text { Ventilação mecânica não } \\
\text { invasiva }\end{array}$ & $9.798,16(8709,48-10.886,85)$ & $43.009,43(4354,74-762079,50)$ & $<0,01$ \\
\hline Exames de imagem: & $11.510,78(2.022,45-169.834,86)$ & $26.291,85(2.022,45-792.562,68)$ & $<0,01$ \\
\hline Raio-x & $12.134,70(2.022,45-169.834,86)$ & $26.291,85(2.022,45-792.562,68)$ & $<0,01$ \\
\hline $\begin{array}{l}\text { Tomografia } \\
\text { computadorizada }\end{array}$ & $8.709,48(2.022,45-169.834,86)$ & $65.953,52(2.022,45-792.562,68)$ & $<0,01$ \\
\hline Ultrassonografia & $16.179,60(6.067,35-169.834,86)$ & $56.628,60(4.044,90-792.562,68)$ & $<0,01$ \\
\hline Ecocardiografia & $16.799,28(6.067,35-169.834,86)$ & $55.617,38(2.022,45-792.562,68)$ & $<0,01$ \\
\hline \multicolumn{4}{|l|}{ Comorbidades associadas } \\
\hline Doenças respiratórias & $10.499,55(2022,45-169.834,86)$ & $26.291,85(2.022,45-73.6171,80)$ & $<0,01$ \\
\hline Doenças gastrointestinais & $15.241,59(14.157,15-20.224,50)$ & $28.314,30(2.022,45-79.2562,68)$ & $<0,01$ \\
\hline \multicolumn{4}{|l|}{ Diagnóstico da internação } \\
\hline Doenças respiratórias & $10.112,25(20.22,45-40.449,00)$ & $16.179,60(2.022,45-72.9418,95)$ & $<0,01$ \\
\hline
\end{tabular}

comparados com aqueles provenientes das unidades de internação (Figura 2a). Os pacientes que permaneceram internados ao final do período do estudo registraram o maior custo mediano total dentre os desfechos estudados, seguidos pelos que foram a óbito e pelos que receberam alta (Figura 2c). Isso provavelmente se deve ao tempo mediano de permanência de 77,5 dias, 21,5 dias e 10 dias para internação hospitalar, óbito e alta, respectivamente. $\mathrm{O}$ custo mediano total dos pacientes que necessitaram de tecnologias na alta hospitalar também foi elevado (Figura 2c).

\section{Discussão}

Os resultados mostraram que a idade mediana de um ano da amostra refletiu o predomínio de condições congênitas e apontaram para a complexidade dos casos pois observou-se uma significativa carga associada às CCC, expressa pela presença de três ou mais diagnósticos, cenário também observado no programa estadunidense Medicaid ${ }^{14}$. Ressalta-se que aproximadamente a totalidade dos pacientes (97\%) registrou até três internações durante o período do estudo e a literatura indica que uma das principais variáveis preditoras para hospitalizações pediátricas, readmissões e despesas hospitalares são as admissões com duas ou mais $\mathrm{CCC}^{7,15}$.

$\mathrm{O}$ custo da assistência médica dos pacientes com CCC foi elevado quando comparado com os pacientes não-crônicos e, proporcionalmente, aumentou em relação à duração da internação hospitalar como esperado. As doenças respiratórias e metabólicas foram as CCC que geraram os maiores custos. Em nosso estudo, a fibrose cística, uma doença progressiva que afeta muitos sistemas, representou o maior grupo de doenças metabólicas, o que poderia explicar a maior proporção dos custos como já observado em outros países $^{21}$. Pacientes com fibrose cística e outras condições complexas, incluindo aqueles com dependência tecnológica, sobrevivem mais do que no passado e têm necessidades especiais de cuidados de saúde que exigem significativos recursos financeiros, hospitalares e comunitários ${ }^{12,21}$.

Neste estudo, o custo mediano total dos pacientes que morreram foi superior ao observado por aqueles que receberam alta hospitalar, sugerindo padrões diferenciados de cronicidade e complexidade e utilização mais intensiva de recursos de saúde. $\mathrm{O}$ estudo de Miller et al. ${ }^{22}$ corrobora este resultado ao mostrar que o tempo de 
1a)

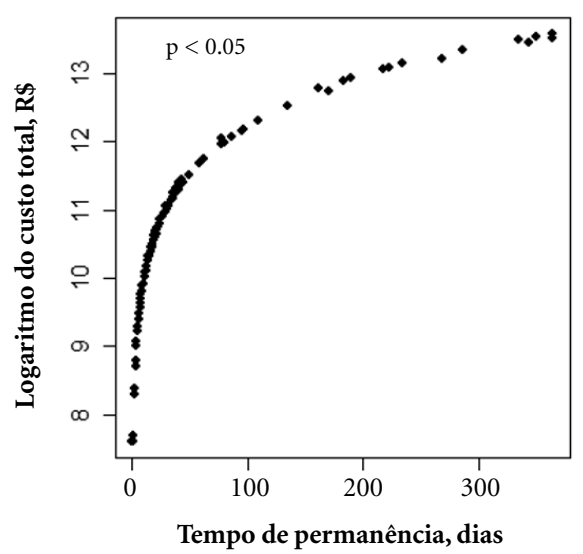

1c)

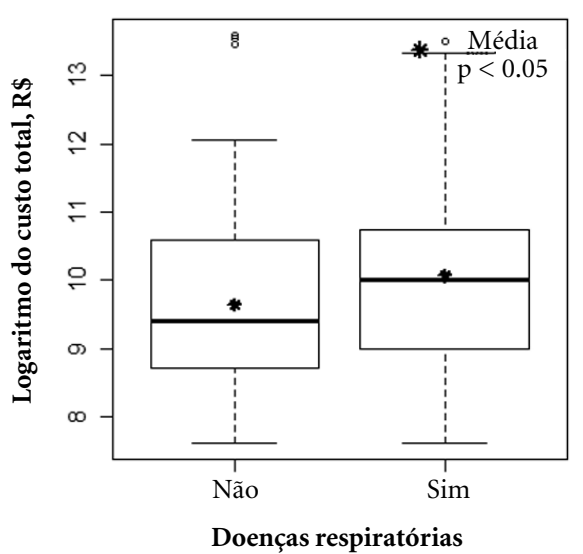

1b)

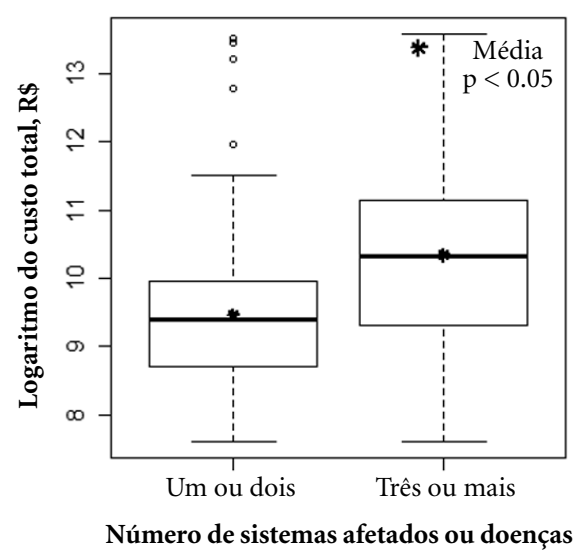

1d)

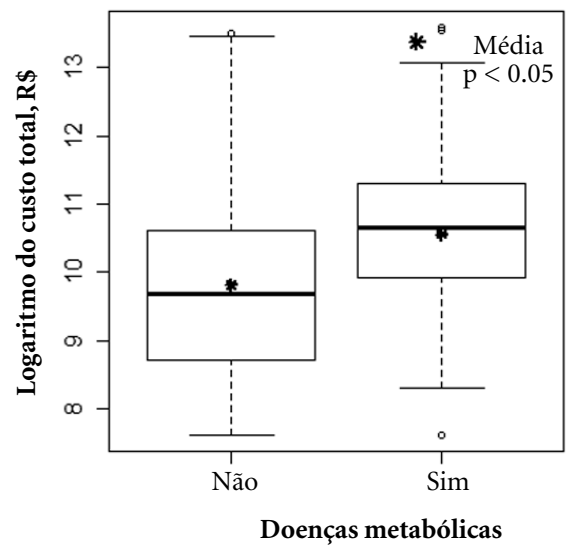

Figura 1. Logaritmo do custo total da atenção hospitalar a paciente com condição crônica complexa de saúde em relação ao tempo de permanência, quantidade de sistemas afetados ou doenças e presença de doenças respiratórias e metabólicas em um hospital público, 2016.

$\mathrm{R} \$$ : reais

estadia de pacientes foi inversamente proporcional à gravidade clínica quando comparados aos pacientes extremamente graves e de gravidade moderada, resultando em maior custo total para os primeiros. Embora nosso objetivo principal não tenha sido estimar o "custo da morte", cabe refletir sobre os desafios e limites que a complexidade clínica impõe e os recursos necessários para enfrentá-los no cenário cotidiano. A expressão “a morte tem um custo" aponta duas questões: os custos para as famílias relacionados às exigências de cuidados abrangentes e permanentes e a obstinação terapêutica dos profissionais de saúde apoiados pelos avanços tecnológicos e terapêuticos. O prolongamento da vida pode requerer me- didas às vezes necessárias para atender às necessidades de alguns pacientes, mas extraordinárias para os outros ${ }^{23}$.

Em relação ao resultado do custo mediano total maior entre pacientes com CCC provenientes das unidades semi-intensiva e de pacientes internados, vale discutir em estudos futuros a trajetória percorrida, que inclui um nascimento em situações adversas que leva à dependência tecnológica e à realização de procedimentos cirúrgicos, prolongando permanências no hospital por anos. Isso se reflete no presente estudo quando se comparou a procedência do paciente, entendida como o lugar por onde entrou na pesquisa, com o resultado do custo total da assistência médica. 
2a)

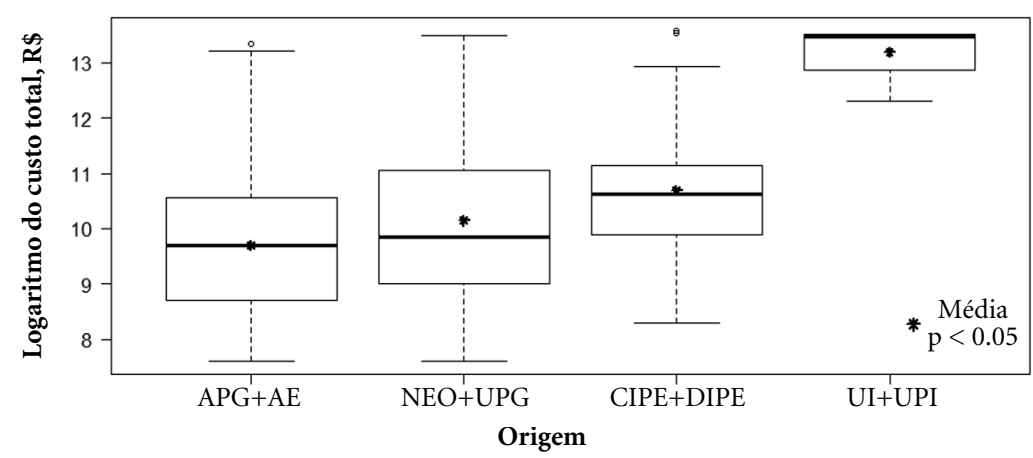

2b)

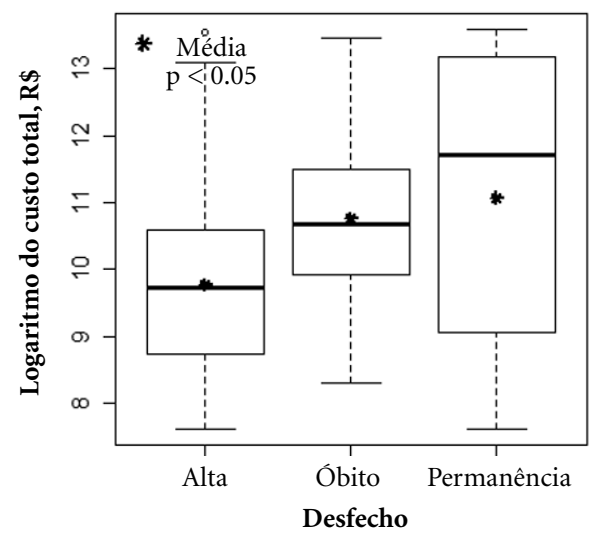

2c)

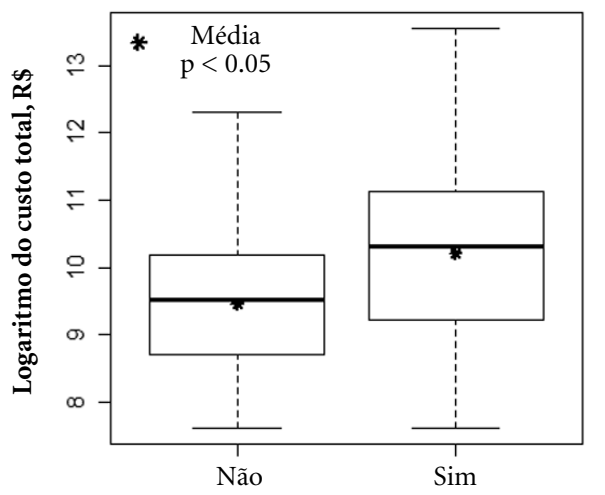

Necessidade de tecnologia na alta hospitalar

Figura 2. Logaritmo do custo total da atenção hospitalar de pacientes com condição crônica complexa em relação à origem, desfecho e necessidade de tecnologia na alta hospitalar.

R\$: reais; APG: ambulatório de pediatria geral; AE: ambulatórios especializados; Neo: unidade de terapia intensiva neonatal; UPG: unidade de terapia intensiva pediátrica; CIPE: enfermaria de cirurgia pediátrica; DIPE: enfermaria de doenças infecciosas; UI: unidade semi-intensiva; UPI: unidade de pacientes internados.

O hospital analisado possui uma unidade semi-intensiva que funciona como unidade de desmame prolongado de ventilação mecânica que impede que os leitos de terapia intensiva pediátrica sejam bloqueados por pacientes com CCC dependentes de ventilação mecânica. Consequentemente, acredita-se que o custo poderia ser ainda maior se os mesmos tivessem permanecido em terapia intensiva, causando impedimento potencial ao acesso de pacientes agudos e instáveis ${ }^{24}$ que se beneficiariam mais desta atenção especializada. Este aspecto merece destaque no que toca à organização de serviços para atenção a esse grupo de pacientes com CCC dependentes de ventilação mecânica. Isso porque induz a articulação e organização da rede de serviços de saúde intra-hospitalar, ambulatorial e de emergência, que se vê confrontada com as necessidades de saúde da população pediátrica no estado do Rio de Janeiro ${ }^{25}$.

Este estudo apresenta algumas limitações. Foi realizado em um único hospital terciário, o que significa que não é possível generalizar os resultados e restringe sua comparação com outros hospitais de menor complexidade. Porém, temse $\operatorname{argumentado}^{7}$ que o hospital é o melhor locus para observar e analisar o número crescente de crianças e adolescentes com CCC, uma vez que este grupo tem frequentes readmissões hospitalares, como também as CCC podem ser desenvolvidas durante o curso de suas doenças. Uma segunda limitação refere-se ao horizonte tempo- 
ral, pois o custo mediano total foi calculado para o período de um ano. Alguns pacientes da coorte já estavam internados antes do início do estudo e a utilização prévia de recursos não foi considerada. Ainda, os pacientes demandam recursos por toda vida e não foram incluídas as consultas ambulatoriais e o custo para as famílias. Este é um componente a ser incorporado em pesquisas futuras, pois são superiores para crianças dependentes de tecnologias ${ }^{15,26-28}$. Ressalta-se ainda que o custo mediano total aumentou de acordo com o número de doenças e sistemas afetados. O nosso objetivo não foi comparar este custo discriminando quais sistemas foram afetados, mas entender como a complexidade poderia impactar na utilização de tecnologias, no tempo de permanência e no custo. Em razão dessas limitações, os resultados podem estar subestimados e sugerese que a pesquisa nesse campo seja ampliada no Brasil a fim de se conhecer o custo sob a perspectiva da sociedade.

Cabe apontar a importância do planejamento das ações de desospitalização na linha de cuidado para esses pacientes, considerando o custo gerado pela dependência tecnológica e as repercussões para o sistema de saúde e para as famí- lias. Agregue-se também outras demandas, como o acesso aos benefícios previdenciários, aos serviços de transporte para tratamento contínuo e fora do domicílio e as adaptações na residência ${ }^{13}$. A programação da desospitalização pode ser um processo complexo com abordagens multidisciplinares, organizadas em etapas que envolvem diferentes níveis de atenção em saúde, a fim de garantir que cada paciente permaneça saudável, se desenvolva e receba suporte para o cuidado contínuo em casa ${ }^{29}$. Além disso, deve-se levar em conta no planejamento da alta a sobrecarga emocional imposta às famílias quando assumem o cuidado cotidiano dos paciente com CCC em casa.

No Brasil, vem se observando ao longo das últimas décadas uma mudança do perfil pediátrico hospitalar ${ }^{25,27}$. Observa-se uma transição para uma "nova pediatria" ${ }^{30}$ que apresenta novos desafios para os profissionais de saúde. À medida que a prevalência de CCC aumenta globalmente, é importante documentar a utilização de recursos de saúde e o custo, ainda que no nível local, bem como entender os obstáculos para planejar de forma eficiente o sistema de saúde para as crianças e adolescentes com CCC.

\section{Colaboradores}

M Pinto: concepção, planejamento do estudo, coleta, análise estatística, interpretação dos dados, redação e revisão do manuscrito. MCN Moreira: concepção, planejamento do estudo, coleta, análise estatística, interpretação dos dados, redação e revisão do manuscrito. RF Tanabe: concepção, planejamento do estudo, coleta, análise estatística, interpretação dos dados, redação e revisão do manuscrito. ACC Costa: coleta, análise estatística, interpretação dos dados, redação e revisão do manuscrito. R Gomes: redação e revisão do manuscrito. 


\section{Referências}

1. World Health Organization (WHO). Innovative care for chronic conditions: building blocks for action: global report. Buildig blocks for action. WHO: Geneva; 2002.

2. McPherson M, Arango P, Fox H, Lauver C, McManus M, Newacheck PW, Perrin JM, Shonkoff JP, Strickland B. A new definition of children with special health care needs. Pediatrics 1998;102(1 Pt. 1):137-140.

3. Cohen E, Kuo DZ, Agrawal R, Berry JG, Bhagat SK, Simon TD, Srivastava R. Children with medical complexity: an emerging population for clinical and research initiatives. Pediatrics 2011;127(3):529-538.

4. Newacheck PW, Kim SE. A national profile of health care utilization and expenditures for children with special health care needs. Arch Pediatr Adolesc Med 2005;159(1):10-17.

5. Simon TD, Berry J, Feudtner C, Stone BL, Sheng X, Bratton SL, Srivastava R. Children with complex chronic conditions in inpatient hospital settings in the United States. Pediatrics 2010;126(4):647-655.

6. Arrue AM, Neves ET, Magnago TS, Cabral IE, Gama SG, Hokerberg YH. Translation and adaptation of the Children with Special Health Care Needs Screener to Brazilian Portuguese. Cad Saude Publica 2016;32(6):e00130215.

7. Burns $\mathrm{KH}$, Casey PH, Lyle RE, Bird TM, Fussell $\mathrm{JJ}$, Robbins JM. Increasing prevalence of medically complex children in US hospitals. Pediatrics 2010;126(4):638-646.

8. Burke RT, Alverson B. Impact of children with medically complex conditions. Pediatrics 2010;126(4):789790 .

9. Castro EK, Piccinini CA. Implicações da doença orgânica crônica na infância para as relações familiares: algumas questões teóricas. 2002. Psicol Reflex Crit 2002;15(3):625-635.

10. Leite MF, Gomes IP, Leite MF, Oliveira BRG, Rosin J, Collet N. Condição crônica na infância durante a hospitalização: sofrimento do cuidador familiar. Ciên Cuid Saude 2012;11(1):51-57.

11. Vieira MA, Lima RAG. Crianças e adolescentes com doença crônica: convivendo com mudanças. Rev Latinoam Enfermagem 2002;10(4):55-60.

12. Hall DE. The care of children with medically complex chronic disease. J Pediatr 2011;159(2):178-180.

13. Moreira MCN, Albernaz LV, Sá MRC, Correia RF, Tanabe RF. Recomendações para uma linha de cuidados para crianças e adolescentes com condições crônicas complexas de saúde. Cad Saude Publica 2017;33(11):1-13.

14. Berry JG, Hall M, Neff J, Goodman D, Cohen E, Agrawal R, Kuo D, Feudtner C. Children with medical complexity and Medicaid: spending and cost savings. Health Aff (Millwood) 2014;33(12):2199-2206.

15. Cohen E, Berry JG, Camacho X, Anderson G, Wodchis W, Guttmann A. Patterns and costs of health care use of children with medical complexity. Pediatrics 2012;130(6):e1463-1470.
16. Brasil P, Calvet GA, Siqueira AM, Wakimoto M, de Sequeira PC, Nobre A, Quintana MS, Mendonça MC, Lupi O, Souza RV, Romero C, Zogbi H, Bressan CS, Alves SS, Lourenço-de-Oliveira R, Nogueira RM, Carvalho MS, Filippis AM, Jaenisch T. Zika Virus Outbreak in Rio de Janeiro, Brazil: Clinical Characterization, Epidemiological and Virological Aspects. PLoS Negl Trop Dis 2016;10(4):e0004636.

17. Brasil. Ministério da Saúde(MS). Centro de Operações de Emergências em Saúde Pública sobre Microcefalias. Monitoramento dos casos de microcefalias no Brasil. Informe Epidemiológico, 33 - Semana Epidemiológica de 26/2016 (26/06 a 02/07/2016). Brasília: MS; 2016.

18. Ananth P, Melvin P, Feudtner C, Wolfe J, Berry JG. Hospital Use in the Last Year of Life for Children With Life-Threatening Complex Chronic Conditions. Pediatrics 2015;136(5):938-946.

19. Feudtner C, Hays RM, Haynes G, Geyer JR, Neff JM, Koepsell TD. Deaths attributed to pediatric complex chronic conditions: national trends and implications for supportive care services. Pediatrics 2001;107(6):E99.

20. Feudtner C, Feinstein JA, Zhong W, Hall M, Dai D. Pediatric complex chronic conditions classification system version 2: updated for ICD-10 and complex medical technology dependence and transplantation. BMC Pediatr 2014;14:199.

21. van Gool K, Norman R, Delatycki MB, Hall J, Massie J. Understanding the costs of care for cystic fibrosis: an analysis by age and health state. Value Health 2013;16(2):345-355

22. Miller RL, Gebremariam A, Odetola FO. Pediatric high-impact conditions in the United States: retrospective analysis of hospitalizations and associated resource use. BMC Pediatr 2012;12:61

23. Diniz D. Quando a morte é um ato de cuidado: obstinação terapêutica em crianças. Cad Saude Publica 2006;22(8):1741-1748.

24. Traiber C, Piva JP, Fritsher CC, Garcia PC, Lago PM, Trotta EA, Ricachinevsky CP, Bueno FU, Baecker V, Lisboa BD. Profile and consequences of children requiring prolonged mechanical ventilation in three Brazilian pediatric intensive care units. Pediatr Crit Care Med 2009;10(3):375-380.

25. Duarte JG, Gomes Júnior SC, Pinto MT, Gomes MAS Perfil dos pacientes internados em serviços de pediatria no município do Rio de Janeiro: mudamos? Physis 2012; 22(1):199-214.

26. Benneyworth BD, Gebremariam A, Clark SJ, Shanley TP, Davis MM. Inpatient health care utilization for children dependent on long-term mechanical ventilation. Pediatrics 2011;127(6):e1533-1541.

27. Costa MT, Gomes MA, Pinto M. Chronic dependence on mechanical pulmonary ventilation in pediatric care: a necessary debate for Brazil's Unified Health System. Ciên Saude Colet 2011;16(10):4147-4159. 
28. Anderson D, Dumont S, Jacobs P, Azzaria L. The personal costs of caring for a child with a disability: a review of the literature. Public Health Rep 2007;122(1):3-16.

29. Elias ER, Murphy NA. Council on Children with D. Home care of children and youth with complex health care needs and technology dependencies. Pediatric 2012;129(5):996-1005.

30. Moreira ME, Goldani MZ. Child is the father of man: new challenges for child health. Ciên Saude Colet 2010;15(2):321-327.

Artigo apresentado em 25/04/2017

Aprovado em 09/04/2018

Versão final apresentada em 11/04/2018 